\title{
Non-invasive ventilation in acute exacerbation of chronic obstructive pulmonary disease: Results from an anonymous survey of physicians
}

\author{
Authors: Chandrashekar Malapanjudi Jagadish, ${ }^{\mathrm{A}}$ Samiksha Gupta ${ }^{\mathrm{A}}$ and Rehan Quadery ${ }^{\mathrm{A}}$
}

\section{Rationale}

Non-invasive ventilation (NIV) is an evidence-based treatment modality for patients with acute exacerbation of chronic obstructive pulmonary disease (COPD) with acute hypercapnic respiratory failure (AHRF). It has been shown to reduce mortality, in-hospital complications, length of stay and is cost effective compared to invasive ventilation. Despite this, data from UK has shown higher mortality and a wide variation in practice in the use of NIV in AHRF.

\section{Aims and objectives}

The aim was to evaluate the knowledge of physicians in the use of NIV in patients with acute exacerbation of COPD in a district hospital setting.

\section{Method}

The study was carried out through means of a voluntary response by physicians (general internal medicine and emergency medicine) at Epsom and St Helier University Hospitals NHS Trust through a standard anonymous online survey (based on British Thoracic Society (BTS) guidelines for the use of NIV in COPD). Respiratory and intensive care physicians were excluded from this study.

\section{Results}

A total of 89 physicians completed the questionnaire, this included $36 \%$ consultants $(n=33), 24 \%$ registrars $(n=21)$ and $40 \%$ senior house officers $(n=36)$. Seventy-five per cent of the doctors had initiated or looked after patients on NIV in the past 12 months. In addition, only $48 \%$ of the doctors had some formal training in the use of NIV. Furthermore, $82 \%$ of doctors were not completely aware of the BTS guidelines in the use of NIV in COPD. Interestingly, $59 \%$ of the respondents felt that they were comfortable in looking after patients on NIV.

\section{Conclusion}

This study showed that a significant number of physicians had managed patients with COPD on NIV, with high confidence levels

Authors: ${ }^{\text {AEpsom }}$ and St Helier University Hospitals NHS Trust, Epsom, UK despite having inadequate knowledge in its use. Furthermore, it highlighted the lack of a formal training in the use of NIV in physicians. The above raises concerns regarding the safety and adequate delivery of NIV in an acute care setting. This, however, is easily remedied with simple education. We recommend that regular training in the use of NIV be organised for physicians who work in acute care setting.

\section{Conflicts of interest}

None declared. 